\title{
Catéchismes et changements culturels
}

\section{Raymond Brodeur}

Volume 56, 1989

Changements culturels et éducation de la foi

Cultural Change and Education of the Faith

URI : https://id.erudit.org/iderudit/1006952ar

DOI : https://doi.org/10.7202/1006952ar

Aller au sommaire du numéro

Éditeur(s)

Les Éditions Historia Ecclesiæ Catholicæ Canadensis Inc.

ISSN

0318-6172 (imprimé)

1927-7067 (numérique)

Découvrir la revue

Citer cet article

Brodeur, R. (1989). Catéchismes et changements culturels. Sessions d'étude -

Société canadienne d'histoire de l'Église catholique, 56, 7-20.

https://doi.org/10.7202/1006952ar

Tous droits réservés @ Les Éditions Historia Ecclesiæ Catholicæ Canadensis Inc., 1989
Ce document est protégé par la loi sur le droit d'auteur. L'utilisation des services d'Érudit (y compris la reproduction) est assujettie à sa politique d'utilisation que vous pouvez consulter en ligne.

https://apropos.erudit.org/fr/usagers/politique-dutilisation/ 


\title{
Catéchismes et changements culturels
}

\author{
Raymond BRODEUR \\ Université Laval, Québec
}

Plusieurs écrits faisant référence aux catéchismes traditionnels, par demandes-réponses, en parlent comme d'ouvrages qui auraient franchi, sans altération, l'histoire. Pourtant, dans son livre L'institution catéchistique au Canada. Deux siècles de formation religieuse (1633-1833), Fernand Porter rapportait une histoire qui, pour la période retenue, comportait quatre générations distinctes de catéchismes. Il est vrai que l'une d'elles, issue de l'arrivée au pays du Catéchisme du diocèse de Sens publié par ordre de $\mathrm{M}^{\mathrm{gr}}$ Languet, prit une importance particulière:

Fait étrange, alors que le catéchisme de $\mathbf{M}^{\mathrm{gr}}$ Languet devait être remplacé, à Sens même, dès 1754, par [celui] de Mgr Paul D'Albert de Luynes, ... il s'introduisait en Nouvelle-France où il devait être le catéchisme qui influença le plus la formation de tout un peuple, et ce, pendant un siècle et demi, période la plus importante de son histoire, 1750-1890 (Porter, 1949: 110-111)1.

Entre l'identification d'un ouvrage qui influença le plus la formation d'un peuple, et l'histoire de la production d'ouvrages spécifiques destinés à faire cette formation, il existe une distance. Mais elle est d'autant plus facile à annihiler, que le jugement de conformité ou d'uniformité rejoint l'opinion que se font les usagers à propos de cette réalité. En effet, des enquêtes orales réalisées par Jeannine Gauthier au cours de l'hiver 1989, dans le cadre d'un volume en préparation sur le fonctionnement du réseau catéchétique québécois de 1888 à 1937, démontrent que tous les informateurs, invités à évoquer leur expérience du livre de catéchisme en paroisse ou à l'école, sont convaincus d'avoir eu en main un ouvrage dont l'origine, le contenu et souvent même la forme «transcendent» les contingences de la culture et des situations socio-politico-économiques qui sont l'apanage des réalités terrestres. À tout considérer, on se retrouve là en face

1 Fernand Porter, L'Institution catéchistique au Canada. Deux siècles de formation religieuse (1633-1833). Montréal, Les éditions franciscaines, 1949, 332p. 
d'un mythe tributaire du phénomène de l'emprise d'une anthropologie religieuse si puissante, si universelle, qu'elle structure, d'une certaine façon, l'esprit critique des individus par rapport à ce qui va en définitive servir de trame de référence, de structure formelle à toute leur existence et à leur identité personnelle et culturelle. Peut-on vraiment qualifier les catéchisés, comme le fit un journaliste montréalais, de zombies? Ou n'est-ce pas un phénomène beaucoup plus complexe qui conduit à reconsidérer le fonctionnement de la culture et de l'identité culturelle dans son rapport au religieux?

La distance chronologique, mais peut-être plus encore psychologique, qui sépare les années 1980 de l'époque des catéchismes traditionnels, favorise une historiographie qui veut de mieux en mieux comprendre les rapports dynamiques entre le religieux et le socio-culturel. Telle est l'orientation du Groupe de recherche sur la production des catéchismes de l'Université Laval, dont le programme global s'énonce ainsi: Pour une histoire de la socio-culture en Amérique française: la production catéchétique. En lien avec les travaux déjà réalisés par le groupe de recherche, et en fonction du thème retenu pour le présent congrès, je me propose, dans un premier temps, d'aborder le catéchisme en tant qu'objet matériel. Cette approche a l'avantage de délivrer le catéchisme de l'emprise d'une anthropologie religieuse afin d'en prendre la mesure, tant dans la quantité que dans la qualité, puis tant dans le temps que dans l'espace. Dans un second temps, en prenant appui sur quelques-uns des constats issus de l'observation, je dégagerai certains éléments relatifs à la production catéchétique, puis à sa distribution et à sa réception. De là, nous pourrons tirer quelques conséquences relatives au thème qui nous rassemble: l'éducation de la foi et les changements culturels.

\section{Le catéchisme comme objet matériel}

La première phase de recherche a consisté à mettre au point un corpus des catéchismes imprimés. Cette enquête fut d'abord amorcée à partir des grandes bibliographies: National Union Catalog, Congress Library, Canadiana, Laurentiana. Mais rapidement, devant la quantité des notices bibliographiques sur les catéchismes, il fallut préciser nos objectifs et ajuster notre méthode de travail. Nous avons été amenés à concentrer nos recherches, non pas sur l'Amérique française, mais sur le Québec.

$\mathrm{Si}$ nous voulions procéder à une histoire de la production des catéchismes, nous ne pouvions nous satisfaire d'une compilation de données bibliographiques de source seconde. Nous devions repérer les ouvrages de première main afin d'y puiser les informations pertinentes à nos futurs travaux, informations que l'on ne retrouve pas nécessairement 
dans les notices bibliographiques: le nom des différents approbateurs, les signataires de liminaires, les noms d'imprimeurs et les lieux d'impression, les grandes divisions de l'ouvrage, etc. Les recherches bibliographiques furent précieuses pour indiquer où se trouvaient certains ouvrages et pour procéder aux vérifications d'usage de nos données en comparaison avec celles de nos prédécesseurs. En raison des différents spécialistes intégrés à l'équipe de recherche, des précisions bibliographiques furent apportées relativement à des auteurs anonymes, à des pseudonymes, ou encore à la datation de certaines éditions, en fonction de la raison sociale des éditeurs/imprimeurs, ou même en fonction de quelques variations du contenu.

Le volume à paraître au cours de l'automne de 1989, s'intitulera Les catéchismes du Québec, (1702-1963). Il présente en tout 957 notices bibliographiques, regroupées en huit catégories différentes:

LES CATÉCHISMES OFFICIELS - Livres, intitulés catéchisme, approuvés et autorisés par l'évêque (ou son tenant lieu) en vue de l'enseignement catéchétique officiel dans son diocèse.

LES CATÉCHISMES EXPLIQUÉS - Livres qui contiennent des explications littérales, des commentaires ou des notes relatives à un catéchisme officiel en usage.

LES CATÉCHISMES SPÉCIFIQUES - Livres qui incluent le mot catéchisme dans leur titre et qui traitent, à l'intention de publics particuliers, d'éléments de doctrine ou de pratiques relatives à la religion.

LES MANUELS D'APPOINT - Livres et documents didactiques qui, bien que non intitulés catéchisme, sont explicitement destinés à parfaire la formation catéchétique, ou à servir à un enseignement religieux.

LES GUIDES D'UTILISATION - Livres destinés aux parents ou aux éducateurs pour indiquer comment se servir d'un catéchisme ou comment l'enseigner.

LES CATÉCHISMES ÉTRANGERS REPRODUITS - Originalement produits et publiés en Europe, ces livres (catéchismes ou manuels d'appoint) ont été réédités ou réimprimés au Québec.

LES CATÉCHISMES ÉTRANGERS UTILISÉS - Originalement produits et publiés en Europe, ces livres (catéchismes ou manuels d'appoint) ont été approuvés par une autorité compétente pour être utilisés au Québec.

LES PSEUDO-CATÉCHISMES - Livres qui incluent le mot catéchisme dans leur titre, mais qui traitent de questions étrangères à la religion. 
Certains ouvrages, en particulier de la catégorie des catéchismes officiels et de la catégorie des catéchismes expliqués, connurent de nombreuses rééditions. D'autres, dont plusieurs dans la catégorie des catéchismes spécifiques, ne furent édités qu'une seule fois. Ces livres qui appartiennent en propre ou par emprunt à une société, une fois repérés et décrits, conduisent à divers constats.

\section{Une chronologie de l'histoire des catéchismes}

Prenant en compte les catéchismes officiels, on rencontre une généalogie d'ouvrages qui se succèdent les uns aux autres. Il faut bien comprendre que, d'après la théologie et dans la pratique, il ne peut jamais exister dans un diocèse qu'un seul catéchisme officiel en usage. Si un évêque décide d'en imposer un nouveau, c'est toujours en excluant celui qui précède. Ainsi, en nous référant à la première édition de chacun des catéchismes officiels, la chronologie présente la périodisation suivante:

1702

Catéchisme du Diocèse de Québec par Mgr de Saint-Vallier

\section{7}

Catéchisme à l'usage du diocèse de Québec

Le Petit catéchisme

Le Grand catéchisme par $\mathrm{Mgr}^{\mathrm{gr}}$ Briand

1815

Le Petit catéchisme du diocèse de Québec

(par Mgr Plessis)
1829

Le Grand catéchisme à l'usage du diocèse de Québec (par Mgr Panet)

par le premier Concile provincial de Québec

1888

Le Catéchisme des provinces ecclésiastiques de Québec, Montréal, Ottawa

par les Archevêques et Évêques de ces provinces

1951

Le Catéchisme catholique

(par une commission épiscopale) 
Cette périodisation correspond à des moments importants de l'évolution de la société. Sans vouloir tomber dans un syncrétisme qui voudrait établir des liens de cause à effet, la chronologie comparée, par exemple, entre les catéchismes officiels et certaines législations montre qu'à chacune des grandes périodes de l'histoire socio-politique correspond, dans l'histoire des catéchismes, la mise en œuvre de mécanismes pour encaisser les transformations qui ont cours et, dans un même mouvement, pour infléchir le sens de ces transformations.

1663: Proclamation royale de la Nouvelle-France

1774: L'Acte de Québec

1791: L'Acte constitutionnel du Canada

1840: L'Acte d'Union

1867: L'Acte de l'Amérique du Nord Britannique

1943: Loi de l'instruction obligatoire
1702: Catéchisme de Saint-Vallier

1777: Catéchisme de $\mathrm{M}^{\mathrm{gr}}$ Briand

1815: Catéchisme de $\mathrm{M}^{\mathrm{gr}}$ Plessis

1854: Catéchisme du Premier Concile provincial de Québec

1888: Catéchisme des Provinces ecclésiastiques

1951: Catéchisme catholique, édition canadienne

\section{Répartition des ouvrages}

Compte tenu de cette périodisation, on peut également opérer une redistribution de l'ensemble des catéchismes et tirer de nouvelles constatations qui intéressent l'histoire de la culture, en particulier en ce qui concerne les transmissions. Par exemple, on constate que jusqu'au milieu du XIX ${ }^{\mathrm{e}}$ siècle, une majorité d'ouvrages viennent de l'Europe. De la fin de ce siècle jusqu'à la Deuxième guerre mondiale, la production locale prend un essor considérable. Toutefois, après cette guerre, on revient à une importation européenne impressionnante, entre autres à la faveur du besoin d'enrichir et de consolider l'enseignement religieux, en particulier auprès des adolescents qui poursuivent de plus en plus leurs études.

\section{La succession des éditions}

Pour ceux qu'intéresse l'histoire de l'imprimé, la réédition de livres de catéchisme est riche d'informations. Elle permet tout d'abord de connaître le réseau des éditeurs imprimeurs qui ont participé à la diffusion de cet ouvrage. Comme le disait Jean-Marie Lebel, du groupe de recherche sur les catéchismes, «si les catéchisés étaient invités à vivre les enseignements de ce livre, les éditeurs eux, devaient vivre de ce livre». 
Du point de vue commercial, le catéchisme officiel était un produit sûr. Si on prend, par exemple, le Petit catéchisme du diocèse de Québec approuvé et autorisé par Mgr Plessis en 1815, on en dénombre actuellement 44 éditions entre 1815 et 1853 . Une étude des mentions d'édition et des mentions de responsabilité intellectuelle ainsi que des approbations inscrites en tête des ouvrages révèle certaines différences. Tous les ouvrages publiés à Montréal jusqu'en 1851 conservent le titre intégral de l'édition de 1815 ainsi que l'approbation signée par Mgr Plessis le premier avril $i \overline{8} 15$. Du côté de Québec, des variations se font remarquer dès 1831. En effet, sur la page titre paraît la mention: Édition revue, examinée et autorisée par $\mathrm{M}^{\mathrm{gr}}$ Bernard-Claude Panet, évêque de Québec. L'approbation signée de $\mathrm{M}^{\mathrm{gr}}$ Panet date du 22 décembre 1830. L'édition de 1834 accorde la responsabilité intellectuelle à $\mathbf{M}^{\mathrm{gr}} \mathrm{Joseph}$ Signay, et cet évêque signera l'approbation le 5 février 1834. En 1837 Mgr Signay fera indiquer comme mention d'édition: édition revue, corrigée et examinée. L'approbation sera datée du $1^{\mathrm{er}}$ avril 1838. L'édition de 1841 aura une nouvelle approbation datée du $1^{\text {er }}$ avril 1841. En 1843, la mention d'édition deviendra: Nouvelle édition autorisée, par $\mathbf{M}^{\mathrm{gr}}$ Joseph Signay, Évêque de Québec. L'approbation sera du 3 janvier 1843. En 1844, l'édition de Cowan reprend les mêmes mentions d'éditions et la même approbation que celles de son édition de 1841, tandis que William Neilson introduit une nouvelle mention d'édition «Édition revue, examinée et autorisée, et une approbation signée par Mgr Signay en date du 15 janvier 1844. En 1845, la mention d'édition des ouvrages parus chez Crémazie et Sinclair devient: Nouvelle édition revue, corrigée et examinée, par ordre de Mgr Joseph Signay, Archevêque de Québec et l'approbation date cette fois-ci du 4 octobre 1845. La même année, les éditions parues chez T. Cary ne mentionnent que «Nouvelle édition autorisée par Mgr Joseph Signay, Archevêque de Québec». L'approbation date du 7 août 1845. Une autre édition faite par T. Cary en 1847 portera la date d'approbation du 14 novembre 18472 .

Ces quelques changements correspondent en réalité à des modifications apportées aux éditions. Dans certains cas, il s'agira de légères variantes relatives au choix des questions marquées d'une astérisque, c'est-à-dire de celles qui sont réservées pour être seules exigées des petits enfants et des «intelligences lourdes». Il pourra s'agir parfois d'un déplacement de jours de jeûne et d'abstinence. Le catéchisme se doit d'être à jour pour la mise en forme de la vie diocésaine.

2 Raymond Brodeur, «Le règne d'un livre: le 〈Petit catéchisme du diocèse de Québec〉 de 1815», Le livre et la lecture au Québec (1800-1850), Claude Galarneau et Maurice Lemire, éd., Québec, Institut Québécois de la recherche sur la culture, 1988, p. 149-165. 


\section{Fixations et variations des contenus}

Autres constats, ceux-là ayant trait aux contenus. D'un ouvrage à l'autre, les sujets traités varient en fonction des objectifs que se donnent les producteurs. Mais également d'une édition à l'autre, des changements apparaissent. Évidemment, d'un certain point de vue, on a raison de dire, comme le font souvent ceux qui regardent l'ensemble du corpus, qu'il n'y a à peu près pas de différence, que c'est toujours la même forme, qu'on retrouve beaucoup de réponses similaires d'un ouvrage à l'autre. Dans un état de science globalisante et d'érudition, il était plutôt hasardeux de vouloir se risquer à aller plus en profondeur. Comme me le disait le père Porter, peu de temps avant sa mort, «vous savez, on travaillait avec les moyens du bord, avec beaucoup de conviction, mais sans grand support matériel». Ce genre de travail d'analyse et de comparaison exige qu'on puisse rassembler des ouvrages, les comparer, les étudier. Sans l'apport des photocopies et des prêts interbibliothécaires permettant facilement une analyse de première main, ces travaux sont impensables. Mais, quand on peut travailler ainsi, les nouvelles pistes de travail fusent. On constate, par exemple, d'une édition à l'autre, l'évolution des couches rédactionnelles. Cela devient précieux pour l'identification précise d'ouvrages rares, mais plus encore pour suivre à la trace l'évolution des univers conceptuels, lesquels sont eux-mêmes tributaires des théologies ou des contextes socioculturels. Par exemple, suivre d'un catéchisme à l'autre les définitions de Dieu, de Jésus-Christ ${ }^{3}$ ou de l'Église ne sont pas sans intérêt, et cela, non pas simplement sur le plan de l'érudition, mais sur l'évolution - ou la fixation - de la manière d'organiser l'univers mental et le cycle annuel. Le catéchisme est donc, d'une certaine manière, fluctuant en fonction de conceptions théologiques personnalisées, des visées pédagogiques particulières et suivant le cycle du calendricr.

\section{Le catéchisme comme objet produit}

Approché comme objet de culture, et non comme compendium de la théologie ne servant qu'à «théologiser», chaque catéchisme et chaque réédition de catéchisme deviennent un produit, et comme tels, suivant l'expression de Bernard Plongeron, «ils fonctionnent selon une ambivalence fondamentale: ce produit est à la fois 〈produit et sproducteur〉 de phénomènes sociaux» ${ }^{4}$.

${ }^{3}$ E. Germain présente, en synopsis une comparaison détaillée du discours sur JésusChrist que véhiculent les catéchismes québécois de 1815, 1854 et 1888. Jésus-Christ dans les catéchismes, Paris, Desclée, 1986, p. 100-110.

4 Bernard Plongeron, «Premier bilan pour de nouvelles approches méthodologiques», Une inconnue de l'histoire de la culture: la production des catéchismes en Amérique française, Québec, Éditions Anne Sigier, 1986, p. 443. 
L'histoire de la production des catéchismes demeure, comme tout ce qui a trait à l'histoire des textes et des imprimés, un lieu fécond pour retracer les diverses tractations et les jeux d'autorités dont procèdent ces derniers. Mais c'est également un espace privilégié pour comprendre les liens qui se tissent, en deçà des cultures particulières, dans une dynamique d'évolution et de diffusion de la pensée (idées et mentalités) qui débordent les frontières géographiques, sans toutefois en faire fi. En matière de culture, le huis clos ou la stagnation sied mal avec des sociétés marquées par les controntations de tous ordres.

Benoît Boily a montré comment, à l'époque de la Nouvelle-France, St. Vallier a produit le Catéchisme du diocèse de Québec en s'inspirant des catéchismes qui l'ont précédé, Fleury, Bossuet...5. Cet ouvrage est remarquable dans sa structure et son contenu. Élisabeth Germain, une éminente historienne des catéchismes, le considère comme un des mieux conçus et elle s'étonne de sa faible longévité. Cet ouvrage produit privément, dans la retraite que s'imposa son auteur, ne lui survécut pas.

Celui de Mgr Briand, en 1777, a une toute autre histoire. Il est une adaptation canadienne d'un des catéchismes français qui souleva le plus de hargne de la part des jansénistes. Les travaux de doctorat de NelsonMartin Dawson ${ }^{6}$, portant sur l'importation du catéchisme de Languet en Nouvelle-France, retracent avec minutie les événements qui entourent la production originale de cet ouvrage. On constate la volonté du producteur de mettre de l'ordre dans une Église qui connaissait certaines dérives, en particulier par rapport à l'autorité magistérielle?

Deux conséquences importantes au plan catéchétique, et par rebond, au plan socio-culturel, se dégagent de l'importation d'un tel livre dans la colonie. Premièrement, au moment où la colonie se prépare à faire face à de profonds bouleversements - la conquête anglaise - la conception de ce catéchisme manifeste les préoccupations pastorales des autorités en place. Une affirmation de la hiérarchie selon la ligne de la stricte orthodoxie s'imposait. Dorénavant, les simples clercs, comme le peuple des fidèles, sont exclus de l'Église enseignante, et relégués dans l'Église enseignée, selon une restriction des catégories, jusqu'ici mal définies. Deuxièmement, cette orientation ne souleva pas dans la colonie les tumultes connus dans la métropole. Dans ce vaste territoire siégeait un seul

5 Benoît Boily, Le Catéchisme du diocèse de Québec, 1702. Son auteur; ses sources, son contenu, Thèse de doctorat présentée à L'Institut Catholique de Paris, mai 1966.

6 Thèse en cours de rédaction à l'Université Laval. Dépôt prévu pour l'automne de 1989.

7 Chantal Van der Planke, Structures de participation et de décisions dans l'Église de France en XVIIIe siècle, en particulier dans la province ecclésiastique de Sens, thèse de doctorat en théologie présentée à l'Université Catholique de Louvain, 1975. 
évêque, choisi par la cour. Son clergé comptait un peu plus d'une centaine de prêtres, les uns bien encadrés par leur maison ecclésiastique, les autres débordés de travail dans leur mission. Dans un tel contexte, les conflits d'intérêts, bien que connus par les élites, ne pouvaient revêtir les mêmes formes ou les mêmes proportions qu'en France. Pour cette société, la conception ecclésiologique centrée sur Rome n'était pas contestée. C'était cette conception que le nouveau catéchisme encadrait plus rigoureusement qu'auparavant. Dawson démontre que dès le début des années 1740 , cet ouvrage fut en usage dans le diocèse de Québec. Ensuite, les premiers libraires canadiens, les protestants Brown et Gilmore, l'éditent à la demande de Louis Germain dit Langlois, marchand général, deux années de suite, soit en 1765 et 1766 . Onze années plus tard, du consentement réciproque de Monsieur Étienne Montgolfier, supérieur du séminaire Saint-Sulpice de Montréal, et de Monseigneur Joseph-Olivier Briand, évêque de Québec, l'ouvrage reçoit une toilette canadienne, colorée par le contexte de pauvreté économique et culturelle des habitants. C'est, en 1777, la double décision de retoucher considérablement l'ancien abrégé du catéchisme et d'autoriser qu'on puisse publier le petit catéchisme séparément du grand.

Quand Plessis prend en charge le diocèse, au début du XIX $\mathrm{X}^{\mathrm{e}}$ siècle, et qu'il a pu régler avec crainte et tremblement la situation politique de son Église dans la colonie, il entreprend une consultation, la première du genre à avoir lieu dans le diocèse, et il produit son catéchisme. Je ne peux pas discuter ici ce que James-H. Lambert a si bien démontré sur le style de pouvoir et d'autorité exercé par Plessis, si ardent à consulter et si soigneux à exécuter ${ }^{8}$. Néanmoins, cet ouvrage, fruit d'une longue expérience pastorale et d'une consultation sérieuse ${ }^{9}$, a été conçu en mettant à profit, en plus d'une demi-douzaine de catéchismes de France, deux catéchismes irlandais, un autre, anglais et un américain: le Douai Catechism et le Butler's Catechism, le Abridgment of Christian Doctrine et le catéchisme de Baltimore. Le pragmatisme anglo-saxon imprègne profondément l'ouvrage. Les questions sont plus courtes, on ne répète plus systématiquement dans la réponse tout ce que vient d'énoncer la question. Soucieux de l'uniformité religieuse de ses ouailles, Plessis voit à faire traduire son catéchisme en anglais, puis en montagnais. Cet ouvrage, riche d'influences diverses, traversera la Rébellion de 1837-1838.

8 James H. Lambert, The Catholic Bishop Joseph Octave Plessis, Church, State and Society in Lower Canada: Historiography and Analysis, Thèse pour l'obtention du Doctorat en Histoire, présentée à l'Université Laval, 1981.

9 Raymond Brodeur, Identité culturelle et identité religieuse: étude d'un cas: "Le petit catéchisme de Québec", approuvé et autorisé par M ${ }^{\text {gr }}$ J.O. Plessis, Québec, le $1^{\text {er }}$ avril 1815 , Thèse de doctorat conjoint en sciences des religions et en théologie présentée à l'Université de Paris IV-Sorbonne et à l'Institut Catholique de Paris, 1982. 
En 1850, les évêques de la jeune Province ecclésiastique de Québec décident, lors du premier concile provincial, la rédaction commune d'un petit catéchisme dans les deux langues. Dans les faits, le catéchisme de Butler sera maintenu pour les catholiques anglais, vu que la plupart de ceux qui parlent cette langue sont presque tous immigrés d'Irlande et y sont accoutumés. Pour les francophones, un nouveau petit catéchisme paraît. Plus volumineux que celui de Plessis, cet ouvrage se présente davantage comme une introduction au grand catéchisme. On revient à l'usage de répéter la question dans la réponse, de manière à ce que ies réponses, coupées des questions, aient tout de même un sens complet en elle-mêmes. De plus, l'ordre de présentation des matières change, plaçant, à l'instar du Catéchisme du Concile de Trente, les sacrements avant les commandements.

Dans la deuxième moitié du siècle, un courant grandit en faveur d'un catéchisme français unique, c'est-à-dire qui intègre le grand et le petit catéchisme. Mais en 1888, les archevêques et suffragants des trois provinces, n'ayant réussi à s'entendre sur un nouveau manuel composé par eux, décident, pour mettre fin à des discussions qui duraient depuis trente ans, $d$ 'adopter un texte qui suit de très près la seconde édition française du Catéchisme promulgué par le troisième concile plénier de Baltimore ${ }^{10}$. Une fois approuvé par les archevêques et suffragants des trois provinces, on entreprend une retraduction anglaise de ce catéchisme.

Cet ouvrage aura un long règne, laissant libre le champ de la créativité dans les autres catégories d'ouvrages à portée catéchétique. En 1941, une commission épiscopale, dirigée par $\mathrm{M}^{\mathrm{gr}}$ Desranleau, entreprendra la tâche de produire un nouveau catéchisme officiel adapté entre autres à la nouvelle situation provoquée par l'instruction obligatoire. Il lui faudra dix années pour aboutir à la production du Catéchisme catholique, édition canadienne, ouvrage qui ne sera jamais traduit en anglais.

Un survol des catéchismes officiels révèle toute une gamme d'imprimés permettant une analyse de l'évolution des cultures. Tandis que certains de ces catéchismes ne sont guère novateurs, d'autres apparaissent comme des instruments sûrs et efficaces de l'évolution de la pensée religieuse du peuple. Que sont ces catéchismes? Sont-ils un outil conceptuel, présentent-ils un idéal à atteindre, sont-ils un guide sécuritaire? Voilà des questions pertinentes à poser à ces produits davantage conçus en fonction du «baptisé catholique à édifier», que du «catholique rencontré au

10 Nive Voisine, «Le catéchisme de 1888: victoire de l'uniformité ou compromis circonstanciel?», Une inconnue de l'histoire de la culture: la production des catéchismes en Amérique française, R. Brodeur et J.P. Rouleau, éd. Québec, Anne Sigier, 1986, pp. 281-302. Élisabeth Germain, Jésus-Christ dans les catéchismes, Paris, Desclée, 1986, p. 100-110. 
quotidien». Plus on travaille à approfondir les questions relatives à «qui produit» les catéchismes, plus on réalise qu'il ne s'agit pas simplement d'histoire anecdotique, mais d'histoire génétique.

\section{Diffuser le livre: une question d'impression!}

Les catéchismes ne sont pas de purs objets religieux pour un Québec simplement religieux, circulant en dehors de toute contingence matérielle. Les travaux actuellement en cours sur le réseau catéchétique québécois (1888-1938) relatent les rapports réciproques entre les livres de catéchismes et les lois du marché de l'imprimé. L'analyse du corpus des catéchismes, sous cet angle, fait ressortir des points communs et des particularités qui colorent le réseau de l'édition québécoise. La filière de l'édition, qui évolue vers une mainmise absolue des maisons d'édition religieuse, a permis à ces livres d'exister selon certaines modalités et à certains prix. On voit se dégager progressivement la variété des éditeurs de catéchismes en fonction de l'évolution des stratégies éditoriales. Ces stratégies concernent évidemment les besoins à combler, mais ces besoins sont euxmêmes largement définis par les diverses autorités impliquées en des lieux divers et habilitées à se prononcer sur le choix des livres, soit pour les imposer ou encore les recommander.

En mettant l'accent sur les catéchismes en tant qu'objets de culture insérés dans l'univers de l'édition, on ne peut éviter de faire la rencontre des éditeurs dans leur temps et leur espace géographique, ayant des préoccupations d'ordre économique. On est en mesure de mieux comprendre le rapport entre les possibilités d'un développement technologique et les capacités de répondre à un marché. On ne peut ignorer, par exemple, les différentes qualités d'éditions possibles et proposées, ainsi que les manœuvres diverses servant à l'obtention des contrats, qu'elles soient de l'ordre de la concurrence entre éditeurs ou des privilèges accordés par les responsables. Pris sous un autre angle, ces travaux permettent de dresser un panorama du réseau de distribution des catéchismes, en indiquant les différents points de vente et de publicité/propagande. Enfin, on obtient certains renseignements sur «la gestion et la rentabilité» de ce commerce.

\section{Diffuser le livre: une question de transmission!}

Une fois distribué, cet ouvrage est enseigné. En règle générale, quelqu'un de formé, d'initié, agit comme guide ou comme intermédiaire pour assurer l'apprentissage intégral du catéchisme. Les travaux en cours veulent rendre compte de ce que signifie la fonction de «catéchiste». Qui sont les catéchistes et quelles sont les diverses modalités d'accomplissement de la fonction qui leur incombe. Dans la signification de la fonction, 
il y a d'abord la mission magistérielle de l'Église, en pointant la distinction entre l'Église enseignante et l'Église enseignée, telle qu'on la repère dans la théologie de l'époque et telle qu'elle se répercute dans une sorte d'organigramme des catéchistes. Pour l'Église, catéchiser est un droit et un devoir découlant de sa nature même. L'exercice de ce droit est encadré dans des lois qui en fixent l'objet et les modalités, en tenant compte des lieux et des temps. Entre les agents de catéchisation s'échelonnent les niveaux de responsabilités, depuis l'évêque, d'abord, puis le prêtre, ensuite ies parents et, enfin, ie personnei enseignant. De pius, on doit étabiir les liens entre les rôles sociaux de chacun de ces agents et leur charge ou responsabilité comme catéchistes. On aborde ici la «compétence naturelle» de chacun et les divers moyens mis en œuvre pour l'émulation à remplir sa tâche. Enfin, concernant les diverses modalités d'accomplissement de la fonction de catéchiste, on pénètre dans «l'art de catéchiser». À cet égard, un nombre considérable d'ouvrages et d'articles destinés à illustrer comment bien faire «la tâche», rend compte de la multiplicité des compétences qui vont de la théologie, à la pédagogie, en passant par la psychologie et le service social. Ces travaux aident à comprendre comment l'organisation qui sous-tend l'acte de catéchiser correspond à une institution globale, et non particulière, porteuse d'un projet de société, et habilitée à instruire des bons Québécois affermis dans leur identité culturelle catholique.

Par ces travaux, on fait le pont entre les catéchismes, objets de culture, et le catéchisme comme agent de culture, porteur et promoteur d'un projet de société. On quitte, d'une certaine façon, le niveau de la description du corpus pour entrer dans l'activité générée par ce corpus. On touche aux personnes dans ce qu'elles sont et ce qu'elles font. Il ne faudra pas perdre de vue que malgré la diversité des catéchistes, il n'y a point parallélisme, mais complémentarité d'interventions. C'est là que la notion d'institution catéchétique prendra son sens le plus fort. C'est une institution imprégnée de culture et imprégnant la culture. Précisément parce qu'elle est présente à tous les niveaux, c'est une institution qui n'a pas de lieu particulier, comme ce sera le cas, après les années 1950, avec l'Office catéchistique québécois.

\section{La réception: les «reçus» du catéchisme}

Que produisent les catéchismes? On est ici face à un dilemme, car c'est vraiment ce qui fait le plus peur aux historiens, alors que tout le monde porte en lui la compétence pour répondre à cette question. En effet, rares sont les québécois qui n'ont pas connu l'expérience du catéchisme. Mais, et c'est bien là la pénible réalité pour des chercheurs épris de la plus 
grande objectivité possible, cette expérience est si mouvante, si insaisissable. Dès sa saisie, elle sort de l'expérience pour devenir discours. Et comble de malheur, ces discours sont rarement produits de la saisie directe de ceux qu'on pourrait nommer les acteurs, mais ils sont habituellement filtrés par l'intermédiaire qui les collige. Problème bien réel qui incite à la nuance et à la prudence.

On ne peut prétendre mettre en boîte de façon irrémédiable la densité des expériences humaines, mais on ne peut se fermer les yeux sur des agirs qui, comme le dit la psychopédagogie, sont expression d'une impression ${ }^{11}$. En effet, les manifestations extérieures sont toujours partie prenante de l'expérience personnelle. Jean Mesny, spécialiste de la pédagogie auprès des enfants, dit que le tout-petit réfléchit en agissant. Dans ce même sens, on peut dire que l'humain réfléchit également en agissant, ou encore que toute action est portée par une réflexion plus ou moins consciente et aussi potentiellement porteuse de prise de conscience. C'est lié au fonctionnement symbolique de l'être humain. C'est en ce sens que l'analyse des manifestations diverses, des expressions multiples des individus, peut permettre d'établir des rapports entre ce qu'est le catéchisme et ce qu'adviennent les catéchisés. Dans le groupe de recherche, nous parlons à ce propos des «reçus du catéchisme».

Les «reçus» du catéchisme sont les traces que celui-ci a inscrites dans les personnes mises en contact avec lui. Pour cela on veut savoir le plus précisément possible, premièrement, qui sont ces catéchisés? Deuxièmement, comment le catéchisme est-il passé dans leur vie? Troisièmement, comment cela a-t-il marqué la suite de leur existence? Et, quatrièmement, qu'en ont-ils conservé tout au long de leur vie? En somme, comment, et dans quelle mesure, le comportement des catéchisés nous permet-il d'attribuer à cette institution culturelle qu'est le catéchisme, les «reçus» qui ont contribué à façonner l'identité personnelle de ces catéchisés?

Pour atteindre cet objectif, nous mettons en exergue les caractéristiques des catéchisés, telles que nous les retrouvons dans certains documents officiels ou encore dans les titres et les textes liminaires des catéchismes. Ensuite, nous illustrons certaines attitudes et comportements de ces catéchisés à partir de témoignages des catéchistes de l'époque, particulièrement dans des rapports de paroisses. Finalement, et surtout, pour la période étudiée, nous laissons la parole aux personnes en recourant à une série de témoignages repérables dans la littérature, ainsi qu'en procédant à des enquêtes orales qui correspondent à nos besoins et à des critères de scientificité ethnologique.

11 Claude Calaguier et Gerard Mallen, Le jeu d'expression et l'imaginaire, Collection pédagogique psychosociale, no. 33, Paris, Fleurus. 


\section{Conclusion}

Nous évoquions au début de cet exposé cette propension généralisée à considérer le catéchisme comme une entité intemporelle, immuablement fixée. Après avoir rapidement considéré la diversité des ouvrages et la multiplicité des énergies déployées pour les produire, les distribuer et les recevoir, on ne peut associer cette opinion générale avec une quelconque «fossilisation», résultat d'un laisser-aller ou d'une banale habitude portée par un courant culturel poursuivant sa trajectoire bon an, mal an. Quand Élisabeth Germain, après avoir procédé à une compilation consciencieuse des textes des catéchismes américains et québécois du XIX ${ }^{\mathrm{e}}$ siècle, demande le pourquoi d'une telle fixité d'un catéchisme ${ }^{12}$, sa réflexion est sous-tendue par une conception de la catéchèse qui, orientée vers l'acte de foi qu'elle tente d'éveiller et de soutenir, devrait davantage coller aux conditions et à l'expérience de vie du croyant. Or, et c'est la question qui se pose, jusqu'où le catéchisme voulait-il catéchiser, du moins en ce sens pastoral? À analyser de près le fonctionnement du réseau catéchétique antérieur à Vatican II, quelle identité d'homme voulait-on mettre en forme et, dans le même mouvement, voulait-on empêcher de prendre forme? En répondant à cette question, on pourra mieux dégager le «Dieu» qu'identifie le catéchisme, un Dieu nécessairement conçu en rapport aux hommes, aimant une Église «fonctionnelle». De là, dit Bernard Plongeron, «on en arrive à la valeur sociale, c'est-à-dire morale, du christianisme» 13. Aussi, quand des gens parlent du catéchisme, se peut-il qu'en réalité ils réfèrent bien davantage à ce qu'a fait fonctionner de façon empirique et symbolique ce catéchisme qu'au catéchisme responsable de ce fonctionnement? Or, si nous voulons comprendre mieux les rapports entre les interventions d'autorité et les changements culturels, entre les projets d'éducation de la foi et l'évolution des cultures, on a avantage à se méfier des jugements hâtifs et à se mettre à l'école des opuscules conçus pour faire école.

12 É. Germain, Jésus-Christ.... op. cit., p. 109.

13 Bernard Plongeron, Théologie et politique au siècle des lumières (1770-1820), Genève, Droz, 1973, p. 307. 